\title{
Matching batterer typologies with their risk of recidivism: Boosting the applicability of typological research
}

José Luis González-Álvarez ${ }^{\mathrm{a}, \mathrm{b}}$, Virginia Soldino ${ }^{\mathrm{c}^{*}}$, Jorge Santos-Hermoso ${ }^{\mathrm{d}}$, and Enrique J. Carbonell-Vayác

${ }^{a}$ Cabinet for Coordination and Studies, Secretary of State for Security. Ministry for Home Affairs, Spain;

${ }^{b}$ Institute of Forensic and Security Sciences. Autonomous University of Madrid (UAM), Spain;

${ }^{c}$ University Research Institute of Criminology and Criminal Science. University of Valencia, Spain;

${ }^{d}$ Department of Biological and Health Psychology, School of Psychology. Autonomous University of Madrid (UAM), Spain.

* virginia.soldino@uv.es Instituto Universitario de Investigación en Criminología y Ciencias Penales. Facultad de Derecho. Edifico Departamental Central / Despacho 1P03. Campus Tarongers s/n E-46071 Valencia (España).

Funding: This research was partially supported by the University Research Institute of Criminology and Criminal Science of the University of Valencia.

Acknowledgments: We would like to thank all members of the Spanish law enforcement agencies, users of the Viogén System, for their meticulous work. 


\title{
Matching batterer typologies with their risk of recidivism: Boosting the applicability of typological research
}

\begin{abstract}
In order to improve the applicability of criminal typologies, Spanish intimate partner violence against women offender subtypes (i.e., high instability/high antisociality, $\mathrm{HiHa}$; low instability/high antisociality, LiHa, high instability/low antisociality, $\mathrm{HiLa}$; low instability/low antisociality, $L i L a$ ) were matched with their police recidivism outcomes in a longitudinal study of 9,672 cases extracted from the VioGén System. Individuals with high antisociality features showed the highest recidivism $(26.5 \% \mathrm{HiHa} ; 22.6 \% \mathrm{LiHa})$ and multirecidivism rates $(10.7 \% \mathrm{HiHa}$; $8.1 \% \mathrm{LiHa})$, and were more likely to be reported for new severe violent episodes against their victims $(8.1 \% \mathrm{LiHa} ; 7.7 \% \mathrm{HiHa}$;) and to violate the protective orders imposed $(28.8 \% \mathrm{HiHa} ; 28.1 \% \mathrm{LiHa})$. Following risk-need-responsivity principles, HiHa and LiHa offenders should be assigned higher intensity treatment programs than LiLa and HiLa individuals. Additionally, the risk posed by all offender subtypes decreased during the follow-up period; however, HiHa offenders presented with the highest risk over time and had a longer police monitoring. Considering this, law enforcement agencies should deploy the most intense police protection measures for victims of HiHa individuals. With regard to prison data, individuals with greater antisociality ( $\mathrm{HiHa}$ and $\mathrm{LiHa}$ ) and criminal versatility (generalist batterers) were the most represented in prison; therefore, it would be advisable to include therapeutic ingredients for common offenders in prisonbased batterer intervention programs.
\end{abstract}

Keywords: intimate partner violence; recidivism; typology; VioGén System; VPR. 
Matching batterer typologies with recidivism risk

\section{Introduction}

According to the World Health Organization (2013), 30\% of women worldwide have suffered physical and/or sexual intimate partner violence (IPV). In Spain, the country where this study was conducted, the last macro survey on Violence Against Women (Delegación del Gobierno contra la Violencia de Género, 2020) found 14.2\% of women in the country had suffered physical and/or sexual IPV, and 31.9\% psychological IPV, at some point in their lives. The physical, psychological, and social consequences of intimate partner violence against women (IPVAW) on primary victims, their families and the wider community have been widely acknowledged (Campbell, 2002; Ellsberg et al., 2008; Guedes et al., 2016; Loxton et al., 2017; Trabold et al., 2020; Vilariño et al., 2018; Vives-Cases et al., 2011), and researchers, professionals, and public administrators are calling for more effective three-level prevention strategies (Ellsberg et al., 2015; García-Moreno et al., 2015; Heise, 2011).

Primary interventions target a wide population and tackle underlying risk factors for IPVAW, such as gender inequality (Brantingham \& Faust, 1976; Ellsberg et al., 2015). Secondary interventions focus on early detection of at-risk individuals (i.e., men who display one or more risk factors associated with IPVAW offending). Whereas tertiary interventions seek to reduce offending by reducing known offenders' recidivism risk factors (Brantingham \& Faust, 1976). In Spain, men serving prison sentences for gender-based violence offenses ${ }^{\mathrm{i}}$ can voluntarily decide whether to participate in a batterer intervention program (BIP) in prison, whereas probationers are court-mandated to BIPs in the community as a condition of their probation period. Updated meta-analytic evidence points toward the usefulness of BIPs in decreasing official recidivism rates when compared to non-treated individuals with IPVAW convictions (Cheng et al., 2019); nonetheless, growing agreement highlights the need to improve their effectiveness by identifying and targeting key IPVAW dynamic risk factors (Lila et al., 2019). 
Prior research has pointed toward the heterogeneous nature of IPVAW (Delsol et al., 2003; Dixon \& Browne, 2003; González-Álvarez et al., 2021) and typological approaches to classify batterers have been discussed on the basis of their validity and applicability in professional practice (i.e., tailoring BIPs, risk management procedures, or police and judicial measures; Amor et al., 2009; Cavanaugh \& Gelles, 2005; Holtzworth-Munroe \& Stuart, 1994; Weber \& Bouman, 2020); although their practical use might not be comparable across countries and jurisdictions. Longitudinal studies on classic IPVAW offender typologies (e.g., Holtzworth-Munroe et al., 2003) have supported, to some extent, the stability of batterer typologies over time (i.e., family only [FO]; borderline/dysphoric [BD]; low-level antisocial [LLA]; and generally violent/antisocial [GVA]; Holtzworth-Munroe et al., 2000). After 1.5 and 3-year follow-up periods, the four subtypes continued to differ in their levels of IPVAW severity, with FO and LLA men displaying less severe IPVAW than BD and GVA men. The FO subgroup was the most stable, suggesting that IPVAW would not inevitably escalate over time; moreover, most LLA men who switched groups moved to the FO group as a result of decreasing levels of reported IPVAW and antisociality. One-third remained in the LLA group, and almost one-fourth remained in the GVA over time (most moved to the FO or LLA groups), whereas only one man remained in the $\mathrm{BD}$ group 3 years later. Among those who had the opportunity for continued violence (they maintained contact with their partner), 93\% of GVA, $86 \%$ of BD, $77 \%$ of LLA, and $60 \%$ of FO men engaged in new violent behavior against their partners. Similar results were found by Thijssen and de Ruiter (2011), who observed highest recidivism rates among GVA individuals and lowest rates among FO men (average 27-month follow-up period). This would support the implicit assumption that lower levels of IPVAW are due to dyadic, relationship, environmental or interactional factors (situational aggression among FO individuals), while more severe IPVAW is primarily 
attributed to the man's personal characteristics that reflect biological and intrapersonal factors (stable aggression among GVA men).

Additionally, significant differences in treatment outcomes have been identified among classic IPVAW offender typologies (Clements et al., 2002). After a 15-month followup, GVA and DB individuals were more likely than LLA and FO men to be rearrested for IPVAW (15\% of GVA men, $22 \%$ of DB, $5.5 \%$ of LLA, and $5.5 \%$ of FO). Furthermore, GVA individuals showed higher violent (47\%; including IPVAW) and general recidivism rates (i.e., any recidivism outcome; 62\%) than the other IPVAW offender subtypes. However, despite the long existence of classic IPVAW offender typologies, BIPs adapted to them have hardly ever been implemented (Weber \& Bouman, 2020), which would prevent drawing sound conclusions about their potential utility.

Considering the case of batterer typologies that have been created using risk indicators as clustering variables (e.g., Loinaz, 2014; Thijssen \& de Ruiter, 2011), BIPs effectiveness might be improved by matching interventions to these batterer subtypes (e.g., IPVAW typologies would help identify high-risk subgroups in need for more intensive interventions). Nevertheless, in the case of penitentiary-based samples, the likely overrepresentation of severe IPVAW (associated with longer prison sentences) might lead to a lack of representativeness of the less violent IPVAW offender subtypes (Herrero et al., 2016). In this sense, classical IPVAW typologies might be of little utility when applied to imprisoned male batterers, whereas specific typologies of batterers in prison (e.g., generalist vs. specialist batterers, that would differ in the versatility of their criminal histories; Herrero et al., 2016) would be more functional when adapting BIPs delivered in prison settings.

With regard to risk prioritization and risk management, several standardized measures that facilitate risk classification according to the statistical likelihood of recidivism have been developed for IPVAW offenders (e.g., the Brief Spousal Assault Form for the Evaluation of 
Risk version 2 [B-SAFER], Kropp et al., 2010; the Ontario Domestic Assault Risk Assessment [ODARA], Hilton et al., 2008; the Spousal Abuse Risk Assessment [SARA]. Kropp et al., 1995; and the Violence Risk Screening-Police Version [V-RISK-POL], Roaldset et al., 2017). In Spain, the Organic Act 1/2004 spurred the police and judicial protection for women exposed to IPVAW and, in 2007, the Comprehensive Monitoring System of Gender-Based Violence Cases (VioGén System) was created (González-Álvarez et al., 2018). The VioGén System is a computer application which gathers information on all reported cases of gender-based violence in the country. In Spain, once a woman reports IPVAW, police officers investigate the situation and prepare a detailed report for the judge. Every case is assessed with the Police Risk Assessment tool (Valoración Policial del Riesgo [VPR]; actuarial protocol proven useful to predict and manage risk in cases of gender-based violence; López-Ossorio et al., 2019b) and, according to the risk level detected (i.e., extreme, high, medium, low, or not appreciated), proportional police protection measures are adopted. In addition, the Spanish judges can use this police risk assessment to issue protective orders (Caballé-Pérez et al., 2020). The risk of IPVAW is then reassessed with a second questionnaire, the Police Assessment of Risk Evolution tool (Valoración Policial de la Evolución del Riesgo; VPER), every time a significant incident occurs (such as a new complaint), or in predetermined periods of time (López-Ossorio et al., 2019a). The goal of the VioGén System dual assessment protocol (VPR and VPER) is to promptly identify reported individuals who pose a higher risk to their partners, in order to assign victim protection resources in the most efficient way; additionally, the risk management approach is oriented to the inactivation of the identified dynamic-relational risk indicators.

However, little is known about the utility and applicability of IPVAW offender typologies in increasing the effectiveness of police and judicial work for the prevention of new IPVAW episodes. One of the few works that deals with this question is Petersson and 
Strand's (2020) longitudinal study (28-month mean follow-up period), which describes and compares 628 arrested and non-arrested male IPVAW perpetrators in Sweden, in terms of their individual characteristics (i.e., generally violent [GV] and partner only [PO] violent), and variables related to the IPVAW incident. Results showed that arrested perpetrators were more likely to be reported for severe forms of IPVAW, being assessed by the police postarrest with a higher recidivism risk, and being more likely to be prosecuted for the reported IPVAW incident. Furthermore, among those perpetrators who were arrested, GV perpetrators four times more likely to recidivate than PO violent individuals.

\section{Purpose of the study}

Recently, an empirical Spanish IPVAW offender typology was proposed using a large-scale representative national sample of gender-based violence cases $(n=9,731)$, and using the VPR risk indicators (González-Álvarez et al., 2021). Following this typology, IPVAW offenders might be classified according to their levels of psychological instability (e.g., severe and very serious psychological violence, threats to harm the victim, death threat, aggression escalation, exaggerated jealousy or suspicion of infidelity, controlling behavior, stalking behavior) and antisociality (i.e., indicators related to the aggressor's criminal history). As a result, $40.6 \%$ of the sample was grouped into a low-instability/low-antisociality cluster (labeled as LiLa), $27.5 \%$ presented with high instability but low antisocial features (i.e., HiLa subgroup), 10.5\% of the sample was characterized by low instability but a high presence of antisocial indicators (i.e., LiHa), and a fourth group of individuals with high instability and high antisocial features (i.e., HiHa, 21.4\%) was detected.

The main goal of the current study was to examine whether these four IPVAW offender subtypes had different recidivism rates, and if they differed in the severity of the new IPVAW episodes. To this end, Spanish IPVAW offender subtypes were matched with their recidivism outcomes (including violation of court protective orders) in a longitudinal 
study. The expectation was that the HiHa subgroup, with high levels of instability and antisociality, would present with higher recidivism rates and of a more severe nature than the other subgroups. Conversely, the LiLa subgroup (i.e., low instability and low antisociality), was expected to have the lowest recidivism rate, with the less severe new IPVAW episodes.

Moreover, we were interested in observing the stability of the four IPVAW typologies after a follow-up period, on the basis of their risk evolution over time, as measured by the VPER. Considering that the VioGén System allocates proportional police protection measures based on the risk level detected, it was hypothesized that the risk levels of all subtypes would decrease in the course of time, but perhaps at a different pace: the risk of HiHa individuals would take longer to decline than would be the case for the LiLa subgroup. This study would contribute to our knowledge of the risk posed over time by each offender subtype, which could be helpful in improving the police and judicial protection of IPVAW victims, by the adoption of early protective measures matched to the risk posed by each subgroup (e.g., low-risk IPVAW subtypes would need less surveillance/victim protection than high-risk offender subtypes); which in turn would optimize the scarce resources available.

In addition, some penitentiary data were used to explore the presence of all four IPVAW offender subtypes in prison settings, as well as to identify specific IPVAW prison typologies (i.e., generalist vs. specialist batterers; Herrero et al., 2016). In this regard, an over-representation of the most antisocial subgroups was expected (i.e., LiHa and $\mathrm{HiHa}$ ). Our findings could be useful to implement functional adaptations of BIPs delivered in prison settings for the most representative IPVAW offender subgroups, which could help prevent post-treatment recidivism outcomes.

\section{Method}




\section{Participants}

The current study used a final sample of 9,672 valid cases extracted from the VioGén System (González-Álvarez et al., 2018). In all cases, the offender was a man and the victim a woman. The mean age of the perpetrators was 39.1 years $(S D=12.4$; range $=14-93)$, and they were mostly Spanish nationals (78.5\%). Victims were also mostly Spanish nationals $(75.6 \%)$ and their mean age was 36.1 years $(S D=11.7$; range $=12-72)$. All the cases in this sample were contained in the one used for the Spanish IPVAW typology development study $(n=9,731$; González-Álvarez et al., 2021). The following distribution of IPVAW offender subtypes resulted in the current sample: $\mathrm{LiLa}(n=3,926 ; 40.6 \%)$; HiLa $(n=2,672 ; 27.6 \%)$; HiHa $(n=$ $2,068 ; 21.4 \%)$; and $\mathrm{LiHa}(n=1,006 ; 10.4 \%)$. These percentages were equivalent to those of the development sample.

\section{Procedure}

Police officers investigating a gender-based violence case in Spain use the data of their crime investigation to register and activate the case on the VioGén System. Next, the risk level of each offender is initially assessed with the VPR, in order to deploy proportional victim protection measures. During the time a case is activated, the risk of IPVAW is reassessed with the VPER for risk management purposes. Cases remain activated until no recidivism risk is detected, which may take from a few days to a few years, depending on the case (González-Álvarez et al., 2018).

For this study, we collected all gender-based violence cases registered in the VioGén System between October and December 2016 (first complaints made by female victims), provided by the Secretary of State for Security (Spanish Ministry of Home Affairs). In total, 10,623 cases were extracted on January 2018, resulting in a follow-up period ranging between 13 and 15 months (depending on the date of the initial complaint). All cases analyzed in our study included some sociodemographic information on victims and offenders 
(to describe the sample), as well as all the VPRs and VPERs conducted by the police officers and all the available data regarding compliance with the protective orders (in order to code risk levels and recidivism outcomes). Data extraction was refined, excluding those cases not including a VPR, duplicated cases and outliers, resulting in a total sample of 9,672 valid cases.

To complement this study, in October 2020, the relevant prison data (criminal convictions sentenced to imprisonment) of the 9,672 individuals in the sample, that had been automatically incorporated into the VioGén System from the Penitentiary Information System (SIP; a digital database of correctional reports from the Spanish prisons), were extracted. Information was found on 1,013 individuals (10.5\%) who, at some point, had entered a Spanish prison (excluding those located in Cataloniaii). It was not possible to collect data on those individuals who, despite being convicted of a gender-based violence offense, had their custodial sentences suspended or were serving alternative penalties/measures to prison $^{\mathrm{iii}}$.

\section{Instruments}

The risk of recidivism of all cases included in our sample was assessed using version 4.0 of the VPR and the VPER (the most current version at the time of data extraction). Both are validated police recidivism risk assessment tools (López-Ossorio et al., 2019a). VPR4.0 includes 39 risk indicators, and VPER 4.0 includes 34 risk and 9 protection indicators. There are two types of VPER 4.0: the VPER-S, used to reassess risk in predetermined periods of time (as part of the risk management procedure); and the VPER-C, adapted to reassess risk each time a new significant incident occurs between the perpetrator and his victim (such as a new complaint). Therefore, when a case includes a risk assessment with the VPER-C, it would be indicating that there has been a police recidivism event.

\section{Measures}


The allocation of cases to each of the IPVAW offender subtypes (i.e., LiLa, LiHa, HiLa, HiHa) was retrieved from a previous study (González-Álvarez et al., 2021). To account for recidivism outcomes, two dichotomous variables (yes/no) were created: recidivism, coded positively when the case included (at least) one VPER-C risk assessment (reflecting a new IPVAW episode: physical, sexual, psychological violence or threats towards his victim, including violations of court protective orders); and multi-recidivism, coded positively when more than one VPER-C risk assessment was conducted. Furthermore, a specific variable was created to account for the violation of court protective orders, along with five additional dichotomous variables detailing the characteristics of such violation (physical approach, virtual contact, with physical violence, with psychological violence, and stalking; CaballéPérez et al., 2020). Finally, the severity of the new IPVAW episode was coded dichotomously (severe/not severe) as indicated by the police officers in the first 4 factors of the first VPER-C of each case (i.e., the recidivism event was coded as severe when any of the indicators of physical, sexual, psychological violence or threats was annotated as "severe" or "very severe").

Follow-up time was calculated as the difference between the date of the first complaint and the date when cases were extracted (January 2018). Time in custody (i.e., time spent in prison after the date of the first complaint) was subtracted, so follow-up time represented the individual's opportunity to recidivate while residing in the community. We also calculated time at risk as the difference between the date of the first complaint and the date of the first police recidivism event, subtracting the time spent in prison (when applicable). The evolution of risk levels was analyzed taking into account the successive risk assessments (from the first to the last VPER) conducted for each case. Risk levels were categorized quantitatively (i.e., extreme $=5$; high $=4$; medium $=3$; low $=2$; not appreciated $=1)$. 
Finally, a new variable was created to analyze prison records, coded positively when the offender's criminal record included (at least) one prison admission. Both the number of admissions to prison and the type of crimes for which they had been convicted were taken into account. IPVAW offenders were classified as specialists if all their imprisonments were due to IPVAW convictions, and as generalists if they had been convicted of other types of criminal offenses (criminal versatility).

\section{Data analysis}

First, we conducted a descriptive analysis of all variables for the total sample. Second, Chisquare tests were conducted to analyze the relationship between IPVAW typologies and recidivism outcomes. When $\chi^{2}$ was significant, corrected standardized residuals $(C S R ;<-2 ;>$ 2) were used to determine which observed cells mainly contributed to this significance. Furthermore, Cramér's $V$ was used as the effect size measure ( $V$ values of .10, .30, and .50 were considered small, moderate, and large effect sizes, respectively; Cohen, 1988). Due to violations of the normality assumption, non-parametric Kruskal-Wallis and Mann-Whitney $U$ tests were conducted for the analysis of quantitative variables (e.g., number of prison records). Bonferroni adjusted pairwise comparisons were used to investigate differences among IPVAW offender subtypes.

As there was no fixed follow-up time for the sample, differences in police recidivism rates among the four subgroups were examined in two steps. First, log-rank Kaplan-Meier method with log-rank (Mantel-Cox) test for significance was employed to explore the notion that the four groups recidivated at equal rates at the follow-up period. All statistical analyses were performed using SPSS Version 21 statistical software Package.

\section{Results}

\section{Police recidivism}


The mean duration of follow-up for this longitudinal study was 1.2 years $(S D=0.11$; range $0-1.33) .144$ individuals (1.5\% of the whole sample) served time in one of the prisons managed by the Spanish General Secretary of Penitentiary Institutions during the follow-up period: $53.5 \%$ where HiHa offenders, $24.3 \% \mathrm{LiHa}, 16.7 \% \mathrm{LiLa}$ and $5.6 \% \mathrm{HiLa}\left(\chi^{2}[3, N=\right.$ $9672]=143.963, p=.000, V=.12)$. Of these, seven individuals (5 HiHa and $2 \mathrm{LiHa}$ ) were in custody during the whole follow-up period, and thus they did not have the opportunity to recidivate while residing in the community. Therefore, all subsequent analyses of recidivism variables were performed excluding such individuals $(n=9,665)$.

First, $25.3 \%$ of cases $(n=2,444)$ did not include any VPER risk assessment (neither VPER-S nor VPER-C). This was due to an immediate inactivation of the case in the VioGén System, as a result of cessation of subsequent police or judicial measures (i.e., risk of recidivism was not estimated and/or the suspect was acquitted). The absence of VPER risk assessments was statistically related to the typology of the offender $\left(\chi^{2}[3, N=9,665]=\right.$ 946.11, $p=.000, V=.31):$ LiLa cases were less likely to include a VPER than expected (59.3\%), whereas $\mathrm{HiHa}(90.6 \%)$ and HiLa cases $(85.8 \%)$ included more than expected. No statistically significant differences were found for the LiHa subgroup (72.8\%).

Second, 1,717 cases included (at least) one VPER-C risk assessment, meaning the IPVAW recidivism rate for an average follow-up of 1.2 years (in days: $M=438.9 ; S D=38.6$; range $=1-486)$ was $17.8 \%$. IPVAW repeat offenders took, on average, 132.7 days to reoffend $(S D=110.31$; range $=0-458$; those individuals with zero days at risk were reported again the same day of their index arrest). Among these cases, 551 (5.7\% of the total sample) included more than one VPER-C risk assessment, and they were labeled as multi-recidivism cases. In 465 cases (4.8\%), the new reported incident was labeled as "severe". Most of these severe new IPVAW incidents were observed in multi-recidivism cases $\left(\chi^{2}[1, N=1,717]=\right.$ $69.72, p=.000, V=.20)$. 
IPVAW recidivism rates for each offender typology are reported in Table 1.

Statistically significant differences were found among IPVAW offender subtypes (albeit with a small effect size) with regard to recidivism variables. HiHa and LiHa individuals were more likely to be reported for new IPV episodes (single and multiple) against their victims, and to use severe violence (more likely among LiHa than among HiHa offenders). In contrast, the LiLa subgroup showed the smallest percentage of recidivism variables, followed by the HiLa subgroup.

[Insert Table 1 about here]

The follow-up period for each case was significantly different depending on the typology of the offender $(\mathrm{H}[3,9672]=12.545, p=.006)$ : HiLa cases were the ones with the longest follow-up period $(M=441.70 ; S D=29.85$; range: $1-486)$; conversely, HiHa cases had the shortest follow-up time $(M=435.26 ; S D=50.64$; range: $1-486$; Bonferroni-adjusted alpha level of 0.008). Nonetheless, the log-rank Kaplan-Meier survival estimator confirmed that, controlling for individual differences in follow-up time, the survival rates of the four IPVAW offender subtypes differed significantly for any new reported IPVAW incident $\left(\chi^{2}[3\right.$, $N=9,665]=220.897, p=.000 ;$ see Figure 1). No statistically significant differences were detected among IPVAW offender subtypes for the amount of time repeat offenders $(n=$ 1,717 ) took to reoffend.

[Insert Figure 1 about here]

\section{Protective Orders}


In our sample, $49 \%$ of victims $(n=4,732)$ enjoyed a court order of protection (i.e., restraining orders and/or prohibition of communication with the victim). Such protective orders were distributed differently across groups $(\chi 2(3, N=9,672)=582.9, p=.000 ; V=.245)$ : HiHa $(67 \% ; C S R=18.5)$ and HiLa cases $(55.1 \% ; C S R=7.4)$ accumulated the highest percentages, in contrast with $\mathrm{LiHa}(47.2 \% ; C S R=-1.2)$ and LiLa cases $(35.8 \% ; C S R=-21.4)$. In relation to recidivism, details on the percentage of protective order violations and the type of such violations are shown in Table 3. In this regard, statistically significant differences were detected among IPVAW offender typologies, although with small effect sizes associated. The highest proportion of protective order violations occurred in $\mathrm{HiHa}$ and $\mathrm{LiHa}$ cases; furthermore, these offenders were more likely to be arrested for approaching the victim. HiHa offenders were more likely to virtually contact their victims than the other subtypes, whereas LiHa individuals stood out for displaying physical violence after the breach of the order.

[Insert Table 2 about here]

\section{Risk levels and evolution of cases}

At the time of a first complaint, all cases in the VioGén System are assessed with the VPR tool, resulting in five police recidivism risk levels, which were distributed significantly differently (medium effect size) across the IPVAW offender typologies (see Table 2). The HiHa cluster accumulated the higher proportion of cases with the three highest risk levels (6\% extreme, $20.1 \%$ high, and $45.1 \%$ medium); followed by the HiLa subgroup, with a majority of individuals classified in the medium (36.7\%) and low risk levels (49.3\%). LiHa individuals stood out for their low risk level (52.7\%), and no risk was appreciated in $70.6 \%$ of LiLa offenders. No cases were coded as extreme in the LiHa and LiLa subgroups, and no high-risk cases were detected among the LiLa offenders. 
[Insert Table 3 about here]

During the time a case is active in the VioGén System, police officers use the VPER tool to follow the impact of the protection measures over the recidivism risk. In this sense, the total number of VPER risk assessments and their resulting risk levels give us a sense of the evolution of risk in the cases analyzed: a large number of VPER risk assessments and high risk levels over time indicate that the offender persists in his violent behavior towards his victim. In our sample, during a follow-up period ranging between 13 and 15 months, the average number of VPER risk assessments conducted per case was 4.03 (range $=0-51)$. In the specific case of VPER-C risk assessments (conducted after a new relevant incident), the average number was 0.27 (range $=0-14)$. Taking into account each IPVAW offender typology, the higher amount of VPER risk assessments was observed among HiHa cases ( $M$ $=5.91 ;$ range $=0-32)$, followed by HiLa $(M=4.58$; range $=0-51), \mathrm{LiHa}(M=4.06$; range $=$ $0-30)$, and, finally, the LiLa typology $(M=2.66$; range $=0-23)$. These differences were statistically significant $(H[3]=1078.546, p=.000)$, as confirmed by all post hoc pairwise comparisons (Bonferroni-adjusted alpha level of 0.008).

Resulting risk levels were coded quantitatively (from extreme $=5$, to not appreciated $=1$ ), and the mean risk level for each IPVAW offender typology was observed after a new VPER risk assessment was conducted. As shown in Figure 2, risk levels of all typologies decreased over time. Furthermore, differences in average risk levels over time were appreciated among typologies: HiHa cases were coded as those with the highest risk over time, followed by LiHa and HiLa cases; conversely, at all times, LiLa cases were associated to the lowest risk levels. 
[Insert Figure 2 about here]

\section{Prison data}

Prison records were detected in 1,013 offenders (10.5\%; number of prison records: $M=3.46$; $S D=3.16$; range $=1-34)$. Both HiHa $(21.3 \% ; C S R=18.1)$ and LiHa offenders $(22.1 \%$; CSR $=12.7)$ were more likely to have been imprisoned than $\operatorname{LiLa}(5.9 \% ; C S R=-12)$ and HiLa offenders $(4.4 \% ; C S R=-12) ;(\chi 2(3, N=9,672)=592.4263, p=.000 ; V=.25)$. Statistically significant differences were also found among IPVAW offender typologies in the number of prison admissions: $\mathrm{HiHa}(M=3.82)$ and $\mathrm{LiHa}(M=3.76)$ offenders had a greater number of prison records than HiLa $(M=2.81)$ and $\operatorname{LiLa}(M=2.84)$ offenders $(H(3)=17.703, p=$ .001). Such differences were confirmed by post hoc pairwise comparisons (Bonferroniadjusted alpha level of 0.008).

The type of offense for which they had been sentenced to prison was specified in 981 cases $(10.14 \%$ of the total sample). In these cases, the offenders were classified into two groups: 305 offenders (31.1\%) were labeled as specialist batterers (SB; i.e., all their imprisonments were due to IPVAW convictions), and $676(68.9 \%)$ as generalist batterers (GB; i.e., they had been convicted of other types of criminal offenses, including IPVAW offending). Results showed that $57.5 \%$ of HiLa offenders were SB $(C S R=6.5)$, whereas $76 \%$ of $\mathrm{LiHa}(C S R=2.6)$ and $73.5 \%$ of $\mathrm{HiHa}(C S R=2.7)$ were $\mathrm{GB}\left(\chi^{2}(3, N=981)=46.665, p=\right.$ $.000 ; V=.22)$. In the case of LiLa offenders, there were no significant differences in the percentages of SB or GB.

\section{Discussion}

The main purpose of this study was to match Spanish IPVAW offender typologies (i.e., LiLa, LiHa, HiHa, HiLa; González-Álvarez et al., 2021) with their risk of recidivism, in order to 
improve the applicability of this empirically derived typology. Reported results showed that, during the follow-up period ( $M=1.2$ years), the police IPVAW recidivism rate in our sample $(n=9,665)$ was $17.8 \%$; furthermore, the multi-recidivism rate was 5.7\%. As expected, statistically significant differences were found among IPVAW offenders in terms of their risk of recidivism, when they were classified according to the Spanish IPVAW offender typologies (González-Alvarez et al., 2021). The results obtained may assist in the improvement of police work preventing new IPVAW and could be used to inform BIP treatment intensity decisions for each typology.

First, results from the analysis of IPVAW police recidivism outcomes confirmed our hypothesis: IPVAW offenders with high levels of instability and antisociality (i.e., HiHa typology) showed the highest recidivism (and multi-recidivism) rates (26.5\%), followed by those with low levels of instability and high levels of antisociality (i.e., LiHa typology; 22.6\%). Likewise, such IPVAW offender subtypes were more likely to be reported for new severe IPVAW episodes against their victims $(8.1 \% \mathrm{LiHa} ; 7.7 \% \mathrm{HiHa})$ than the other two subtypes. Furthermore, these offenders were also more likely to violate the protective orders imposed (28.8\% $\mathrm{HiHa} ; 28.1 \% \mathrm{LiHa})$, and in such cases, the highest proportion of physical violence toward their victim occurred in LiHa cases (20.3\%). Conversely, IPVAW offenders with low levels of instability and antisociality (i.e., LiLa typology) showed the lowest recidivism rates (11.9\%). These results align with Thijssen and de Ruiter's (2011) prior findings (i.e., highest recidivism rates among GVA individuals) and highlight the importance of the antisocial dimension of IPVAW offending on recidivism. Taking into account the need to match offenders' risk levels to the intensity of BIPs (Bonta \& Andrews, 2017), it would be suitable to apply the highest intensity of treatment to HiHa and LiHa offenders.

Given that the Spanish IPVAW offender typologies were created incorporating some IPVAW risk indicators related to the recidivism of the offenders, correspondence was found 
among the risk levels estimated by the VPR risk assessment and the typologies. HiHa offenders were labeled as those with the highest recidivism risk, followed by HiLa, LiHa and LiLa offenders. In this sense, it would be appropriate to warn the police officers in charge of the protection of the victims of the risk presented by such offender typologies, for example, by incorporating an alert into the VioGén System when a case is coded as HiHa. It could also be useful to warn victims of the profile of their aggressors, in order to increase self-protection measures in the most dangerous cases, paying special attention to critical moments for recidivism, considering new IPVAW incidents occurred, on average, between 4 and 5 months after the first complaint.

In relation to the stability of the risk posed by IPVAW offenders, it was predicted that all typologies would reduce their risk levels as a consequence of the adoption of police protection measures. According to the hypothesis of the study by Holtzworth-Munroe et al. (2003), FO offenders would present a greater decrease in their risk levels than DB and GVA subtypes. According to our results, all the typologies showed a lower risk level over time (follow-up period ranging between 13 and 15 months). Specifically, HiHa offenders (corresponding to the DB and GVA subtypes) were coded as those with the highest risk over time and had a longer police follow-up (highest number of VPER risk assessments), followed in descending order by the HiLa (i.e., DB), LiHa (i.e., GVA) and LiLa (i.e., FO) typologies. Despite observing that some offenders recidivated during the follow-up period, the fact that none of the typologies showed an increase in their risk levels confirms the usefulness of the early adoption of police protection measures in IPVAW cases, and, especially, the long-term maintenance of this type of measures in the cases that are more resistant to change (i.e., HiHa cases).

With regard to prison data, the first result to highlight is that only $10.5 \%$ of the sample had a prison record. In accordance with the results outlined above, the distribution of IPVAW 
offenders in prison was also different depending on their typology: both $\mathrm{HiHa}(21.3 \%)$ and LiHa offenders $(22.1 \%)$ were more likely to have served time in prison than LiLa (5.9\%) and HiLa individuals (4.4\%). Taking into account that those individuals with greater antisociality were the most represented in prison, we were interested to know if said antisociality was related to criminal versatility or, on the contrary, they were specialized IPVAW offenders. As expected, most $\mathrm{LiHa}(76 \%)$ and $\mathrm{HiHa}(73.5 \%)$ inmates were labeled as GB. Given that more violent and versatile IPVAW offenders seem to be overrepresented in prison settings (two thirds of IPVAW offenders in prison had been convicted of other types of criminal offenses; Herrero et al., 2016; Holtzworth-Munroe \& Stuart, 1994), it would be advisable for the therapists in charge of the BIPs delivered in prisons to also work on the criminogenic needs of the inmates that are not directly related to IPVAW, for example, including them in treatment groups for common offenders. In this regard, an analysis of antisociality measures or at least the criminal history of these individuals would provide key information.

\section{Limitations and future directions}

A major concern for research based on police data is that the information gleaned from law enforcement agencies may not represent the full extent of any offending (i.e., the police cannot assume that no incriminating information has been hidden, deleted, or remains otherwise undetected), especially when investigating IPVAW. According to recent data, only $21.7 \%$ of IPVAW victims in Spain have reported their aggressor (Delegación del Gobierno contra la Violencia de Género, 2020), which would prevent a detailed analysis of the characteristics of those unreported cases that are not registered in the VioGén System. On the other hand, taking into account the presumption of innocence of the individuals arrested by the police, some of the cases incorporated into the VioGén system may not end in a conviction (according to the Spanish State Attorney General's Office report, 71.3\% of IPVAW defendants tried in 2019 were convicted; Fiscalía General del Estado, 2020), and 
thus, would no longer be considered as IPVAW cases. However, and unlike most of the published studies on IPVAW offenders that inform as limitations the use of relatively small, non-representative samples; the current sample represents, to date, the largest and most representative sample used in typological studies, which allows us to reach sound conclusions.

Taking into account potential intercultural differences in the profile of IPVAW offenders, we must highlight that our study was carried out with a Spanish sample, classified according to the Spanish IPVAW offender typology (González-Álvarez et al., 2021). In this sense, we encourage new large-scale empirical typological studies in geographically and culturally different samples, which might provide new evidence about the cross-cultural validity of this suggested classification.

\section{Conclusion}

This study offers empirical evidence of the potential utility of the Spanish IPVAW offender typology (González-Álvarez et al., 2021) in forensic settings. First, it provides evidence on how to tailor BIPs to offenders presenting with different degrees of risk and with specific criminogenic needs, and supports prior criticism on 'one-size-fits-all' BIP's approaches (Travers et al., 2021). Specifically, the findings of the present study support the allocation of HiHa and LiHa IPVAW offenders to higher intensity treatments. Furthermore, taking into account the criminal versatility of IPVAW offenders who enter prison, it is recommended that BIPs delivered in prison settings address criminogenic needs not specifically related to IPVAW. Second, in relation to the police protection of victims, it is recommended to incorporate into the VioGén System algorithms that classify reported individuals into the four IPVAW offender typologies, in order to improve risk assessment and flag HiHa cases as those who pose the greatest risk to victims. 


\section{References}

Amor, P. J., Echeburua, E., \& Loinaz, I. (2009). ¿Se puede establecer una clasificación tipológica de los hombres violentos contra su pareja? [Is it possible to establish a typological classification of violent men against their partner?] International Journal of Clinical and Health Psychology, 9(3), 519-539.

Bonta, J., \& Andrews, D. A. (2017). The psychology of criminal conduct (6th ed.). Routledge. Brantingham, P. J., \& Faust, F. L. (1976). A conceptual model of crime prevention. Crime \& Delinquency, 22(3), 284-296. https://doi.org/10.1177/001112877602200302

Campbell, J. C. (2002). Health consequences of intimate partner violence. The Lancet, 359(9314), 1331-1336. https://doi.org/10.1016/S0140-6736(02)08336-8

Cavanaugh, M. M., \& Gelles, R. J. (2005). The utility of male domestic violence offender typologies. Journal of Interpersonal Violence, 20(2), 155-166.

https://doi.org/10.1177/0886260504268763

Caballé-Pérez, M., Villalba-García, D., Santos-Hermoso, J., López-Ossorio, J. J., \& González-Álvarez, J. L. (2020). El quebrantamiento de las órdenes de protección en violencia de género: análisis de los indicadores de riesgo mediante el formulario VPR4.0 [Breach of protection orders in gender-based violence: analysis of risk indicators using the VPR4.0 form]. Anuario de Psicología Jurídica, 30, 63-72. https://doi.org/10.5093/apj2019a17

Cheng, S.-Y., Davis, M., Jonson-Reid, M., \& Yaeger, L. (2019). Compared to what? A metaanalysis of batterer intervention studies using nontreated controls or comparisons. Trauma, Violence, \& Abuse, advance online publication. https://doi.org/10.1177/1524838019865927

Clements, K., Holtzworth-Munroe, A., Gondolf, E., \& Meehan, J. (2002). Testing the 
Holtzworth-Munroe et al. (2000) batterer typology among court-referred maritally violent men. Poster Presented at the Annual Meeting of the Association for the Advancement of Behavior Therapy (2002, November).

Delegación del Gobierno contra la Violencia de Género. (2020). Macroencuesta de violencia contra la mujer 2019 [Macro-survey of violence against women 2019].

https://www.lamoncloa.gob.es/serviciosdeprensa/notasprensa/igualdad/Documents/2020 /110920-Macroencuesta2019_Estudio.pdf

Delsol, C., Margolin, G., \& John, R. S. (2003). A typology of maritally violent men and correlates of violence in a community sample. Journal of Marriage and Family, 65(3), 635-651. https://doi.org/10.1111/j.1741-3737.2003.00635.x

Dixon, L., \& Browne, K. (2003). The heterogeneity of spouse abuse: A review. Aggression and Violent Behavior, 8(1), 107-130. https://doi.org/10.1016/S1359-1789(02)00104-0

Ellsberg, M., Arango, D. J., Morton, M., Gennari, F., Kiplesund, S., Contreras, M., \& Watts, C. (2015). Prevention of violence against women and girls: What does the evidence say? The Lancet, 385(9977), 1555-1566. https://doi.org/10.1016/S0140-6736(14)61703-7

Ellsberg, M., Jansen, H. A., Heise, L., Watts, C. H., \& Garcia-Moreno, C. (2008). Intimate partner violence and women's physical and mental health in the WHO multi-country study on women's health and domestic violence: an observational study. The Lancet, 371(9619), 1165-1172. https://doi.org/10.1016/S0140-6736(08)60522-X

Fiscalía General del Estado (2020). Memoria de la Fiscalía General del Estado [State Attorney General's Office report]Madrid: Fiscalía General del Estado. Ministerio de Justicia.

García-Moreno, C., Zimmerman, C., Morris-Gehring, A., Heise, L., Amin, A., Abrahams, N., Montoya, O., Bhate-Deosthali, P., Kilonzo, N., \& Watts, C. (2015). Addressing violence against women: A call to action. The Lancet, 385(9978), 1685-1695. 
https://doi.org/10.1016/S0140-6736(14)61830-4

González-Álvarez, J. L., López-Ossorio, J. J., Urruela, C., \& Rodríguez-Díaz, M. (2018).

Integral monitoring system in cases of gender violence. VioGén System. Behavior \& Law Journal, 4(1), 29-40.

González-Álvarez, J. L., Santos-Hermoso, J., Soldino, V., \& Carbonell-Vayá, E. J. (2021).

Male perpetrators of intimate partner violence against women: A Spanish typology.

Journal of Interpersonal Violence, advance online publication.

https://doi.org/10.1177/0886260521997442

Guedes, A., Bott, S., Garcia-Moreno, C., \& Colombini, M. (2016). Bridging the gaps: A global review of intersections of violence against women and violence against children. Global Health Action, 9(1), 31516. https://doi.org/10.3402/gha.v9.31516

Heise, L. (2011). What works to prevent partner violence? An evidence overview. https://researchonline.lshtm.ac.uk/id/eprint/21062/1/Heise_Partner_Violence_evidence_ overview.pdf

Herrero, J., Torres, A., Fernández-Suárez, A., \& Rodríguez-Díaz, F. J. (2016). Generalists versus specialists: Toward a typology of batterers in prison. European Journal of Psychology Applied to Legal Context, 8(1), 19-26.

https://doi.org/10.1016/j.ejpal.2015.09.002

Hilton, N. Z., Harris, G. T., Rice, M. E., Houghton, R. E., \& Eke, A. W. (2008). An indepth actuarial assessment for wife assault recidivism: The Domestic Violence Risk Appraisal Guide. Law and Human Behavior, 32(2), 150-163. https://doi.org/10.1007/s10979-0079088-6

Holtzworth-Munroe, A., Meehan, J. C., Herron, K., Rehman, U., \& Stuart, G. L. (2000). Testing the Holtzworth-Munroe and Stuart (1994) batterer typology. Journal of Consulting and Clinical Psychology, 68(6), 1000-1019. https://doi.org/10.1037/0022- 
006X.68.6.1000

Holtzworth-Munroe, A., Meehan, J. C., Herron, K., Rehman, U., \& Stuart, G. L. (2003). Do subtypes of maritally violent men continue to differ over time? Journal of Consulting and Clinical Psychology, 71(4), 728-740. https://doi.org/10.1037/0022-006X.71.4.728

Holtzworth-Munroe, A., \& Stuart, G. L. (1994). Typologies of male batterers: Three subtypes and the differences among them. Psychological Bulletin, 116(3), 476-497. https://doi.org/10.1037/0033-2909.116.3.476

Kropp, P., Hart, S., Webster, C. D., \& Eaves, D. (1995). Manual for the Spousal Assault Risk Assessment Guide (2nd ed.). The British Columbia Institute Against Family Violence.

Kropp, P. R., Hart, S. D., \& Belfrage, H. (2010). Brief Spousal Assault Form for the Evaluation of Risk (B-SAFER), version 2: User manual. ProActive ReSolutions.

Lila, M., Martín-Fernández, M., Gracia, E., López-Ossorio, J. J., \& González, J. L. (2019). Identifying key predictors of recidivism among offenders attending a batterer intervention program: A survival analysis. Psychosocial Intervention, 28(3), 157-167. https://doi.org/10.5093/pi2019a19

Loinaz, I. (2014). Typologies, risk and recidivism in partner-violent men with the B-SAFER: A pilot study. Psychology, Crime \& Law, 20(2), 183-198. https://doi.org/10.1080/1068316X.2013.770854

López-Ossorio, J. J., González-Álvarez, J. L., Muñoz Vicente, J. M., Urruela Cortés, C., \& Andrés-Pueyo, A. (2019a). Validation and calibration of the Spanish police intimate partner violence risk assessment system (VioGén). Journal of Police and Criminal Psychology, 34(4), 439-449. https://doi.org/10.1007/s11896-019-09322-9

López-Ossorio, J. J., Loinaz, I., \& González-Álvarez, J. L. (2019b). Protocolo para la valoración policial del riesgo de violencia de género (VPR4.0): revisión de su funcionamiento. [Protocol for the police gender violence risk assessment (VPR4.0): 
Review of its performance]. Revista Espanola de Medicina Legal, 45(2), 52-58. https://doi.org/10.1016/j.reml.2019.01.002

Loxton, D., Dolja-Gore, X., Anderson, A. E., \& Townsend, N. (2017). Intimate partner violence adversely impacts health over 16 years and across generations: A longitudinal cohort study. PLoS ONE, 12(6). https://doi.org/10.1371/journal.pone.0178138

Ministerio del Interior (2020). Anuario Estadístico del Ministerio del Interior 2019 [2019 Annual Statistical Directory of the Ministry of Home Affairs]. Ministerio del Interior: Secretaría General Técnica.

Petersson, J. \& Strand, S. (2020). Characteristics and recidivism in relation to arrest: Differentiating between partner violent perpetrator subtypes. Nordic Journal of Criminology, 21(2), 203-222, https://doi.org/10.1080/2578983X.2020.1727119

Roaldset, J. O., Hartvig, P., \& Bjørkly, S. (2017). Psychometric properties and predictive validity of a police version of a violence risk screen - A pilot study. International Journal of Law and Psychiatry, 54, 133-139. https://doi.org/10.1016/j.ijlp.2017.06.007

Thijssen, J., \& de Ruiter, C. (2011). Identifying subtypes of spousal assaulters using the BSAFER. Journal of Interpersonal Violence, 26(7), 1307-1321. https://doi.org/10.1177/0886260510369129

Trabold, N., McMahon, J., Alsobrooks, S., Whitney, S., \& Mittal, M. (2020). A systematic review of intimate partner violence interventions: State of the field and implications for practitioners. Trauma, Violence, \& Abuse, 21(2), 311-325. https://doi.org/10.1177/1524838018767934

Travers, Á., McDonagh, T., Cunningham, T., Armour, A., \& Hansen, M. (2021). The effectiveness of interventions to prevent recidivism in perpetrators of intimate partner violence: A systematic review and meta-analysis. Clinical Psychology Review, 84, 101974. https://doi.org/10.1016/j.cpr.2021.101974 
Vilariño, M., Amado, B. G., Vázquez, M. J., \& Arce, R. (2018). Psychological harm in women victims of intimate partner violence: Epidemiology and quantification of injury in mental health markers. Psychosocial Intervention, 27(3), 145-152. https://doi.org/10.5093/pi2018a23

Vives-Cases, C., Ruiz-Cantero, M. T., Escribà-Agüir, V., \& Miralles, J. J. (2011). The effect of intimate partner violence and other forms of violence against women on health. Journal of Public Health, 33(1), 15-21. https://doi.org/10.1093/pubmed/fdq101

Weber, T., \& Bouman, Y. H. A. (2020). Intimate partner violence: Variations in perpetrators and treatment allocation. Journal of Interpersonal Violence, 35(3-4), 1012-1034. https://doi.org/10.1177/0886260517692994

World Health Organization. (2013). Global and regional estimates of violence against women: prevalence and health effects of intimate partner violence and non-partner sexual violence.

https://apps.who.int/iris/bitstream/handle/10665/85239/9789241564625_eng.pdf;jsessio nid=0D801E4E8F709E508151EE1BA7C60F21?sequence $=1$ 
Table 1

Recidivism rates for each offender typology $(N=9,665)$

\begin{tabular}{|c|c|c|c|c|c|c|c|}
\hline \multirow{2}{*}{\multicolumn{2}{|c|}{ Recidivism variables }} & \multicolumn{4}{|c|}{ Offender typology } & \multirow[b]{2}{*}{$\chi^{2}$} & \multirow[b]{2}{*}{ Cramer's $V$} \\
\hline & & $\begin{array}{c}\text { LiLa } \\
(n=3,926)\end{array}$ & $\begin{array}{c}\text { HiLa } \\
(n=2,672)\end{array}$ & $\begin{array}{c}\text { HiHa } \\
(n=2,063)\end{array}$ & $\begin{array}{c}\mathrm{LiHa} \\
(n=1,004)\end{array}$ & & \\
\hline \multirow{3}{*}{ Recidivism } & & & & & & \multirow{3}{*}{$214.042 * * *$} & \multirow{3}{*}{.149} \\
\hline & Presence (\%) & 11.9 & 11.8 & 20.3 & 22.6 & & \\
\hline & $C S R$ & -12.4 & 0 & 11.7 & 4.2 & & \\
\hline \multirow{2}{*}{$\begin{array}{l}\text { Multi- } \\
\text { recidivism }\end{array}$} & Presence $(\%)$ & 2.7 & 5.7 & 10.2 & 8.1 & \multirow{2}{*}{$152.087 * * *$} & \multirow{2}{*}{.125} \\
\hline & $C S R$ & -10.4 & 0.1 & 9.9 & 3.4 & & \\
\hline \multirow{2}{*}{ Severe } & Presence $(\%)$ & 3.2 & 3.8 & 7.7 & 8.1 & \multirow{2}{*}{$88.681 * * *$} & \multirow{2}{*}{.096} \\
\hline & $C S R$ & -6.2 & -2.9 & 6.8 & 5.1 & & \\
\hline
\end{tabular}

Note. CSR $=$ corrected standardized residuals; $* p<.05 ; * * p<.01 ; * * * p<.001$. 


\section{Table 2}

Violation of protective orders for each offender typology $(N=4,732)$

\begin{tabular}{|c|c|c|c|c|c|c|c|}
\hline \multirow{2}{*}{\multicolumn{2}{|c|}{ Type of protective order violation }} & \multicolumn{4}{|c|}{ Offender typology } & \multirow[b]{2}{*}{$\chi^{2}$} & \multirow[b]{2}{*}{ Cramer's $V$} \\
\hline & & $\begin{array}{c}\text { LiLa } \\
(n=1,406)\end{array}$ & \multirow{2}{*}{$\begin{array}{c}\begin{array}{c}\text { HiLa } \\
(n=1,472)\end{array} \\
22.8\end{array}$} & \multirow{2}{*}{$\begin{array}{c}\begin{array}{c}\mathrm{HiHa} \\
(n=1,381)\end{array} \\
28.8\end{array}$} & \multirow{2}{*}{$\begin{array}{c}\begin{array}{c}\mathrm{LiHa} \\
(n=472)\end{array} \\
28.1\end{array}$} & & \\
\hline \multirow{2}{*}{$\begin{array}{l}\text { Protective order } \\
\text { violation }\end{array}$} & Presence (\%) & 16.6 & & & & $64.800 * * *$ & .117 \\
\hline & $C S R$ & -7 & -0.5 & 5.8 & 2.6 & & \\
\hline \multirow{2}{*}{ Physical approach } & Presence $(\%)$ & 14.1 & 18.7 & 24.3 & 24.3 & $50.894 * * *$ & .106 \\
\hline & CSR & -6 & -0.9 & 5.2 & 2.7 & & \\
\hline \multirow{2}{*}{ Virtual contact } & Presence $(\%)$ & 3.4 & 6.3 & 9.8 & 5 & $41.902 * * *$ & .104 \\
\hline & $C S R$ & -5 & 0 & 5.8 & -1 & & \\
\hline \multirow{2}{*}{$\begin{array}{l}\text { With physical } \\
\text { violence }\end{array}$} & Presence $(\%)$ & 12.3 & 10.4 & 15.4 & 20.3 & $9.366^{*}$ & .092 \\
\hline & $C S R$ & -0.8 & -2.2 & 1.2 & 2.3 & & \\
\hline \multirow{2}{*}{$\begin{array}{l}\text { With psychological } \\
\text { violence }\end{array}$} & Presence (\%) & 17.1 & 18.8 & 23.2 & 24.1 & 5.071 & .068 \\
\hline & $C S R$ & -1.5 & -1 & 1.6 & 1 & & \\
\hline \multirow{2}{*}{ Stalking } & Presence $(\%)$ & 14.8 & 21.1 & 20.6 & 24.8 & 6.203 & .075 \\
\hline & $C S R$ & -2.3 & 0.6 & 0.4 & 1.5 & & \\
\hline
\end{tabular}

Note. $C S R=$ corrected standardized residuals; $* p<.05 ; * * p<.01 ; * * * p<.001$. 
Table 3

VPR police recidivism risk levels for each offender typology $(N=9,672)$

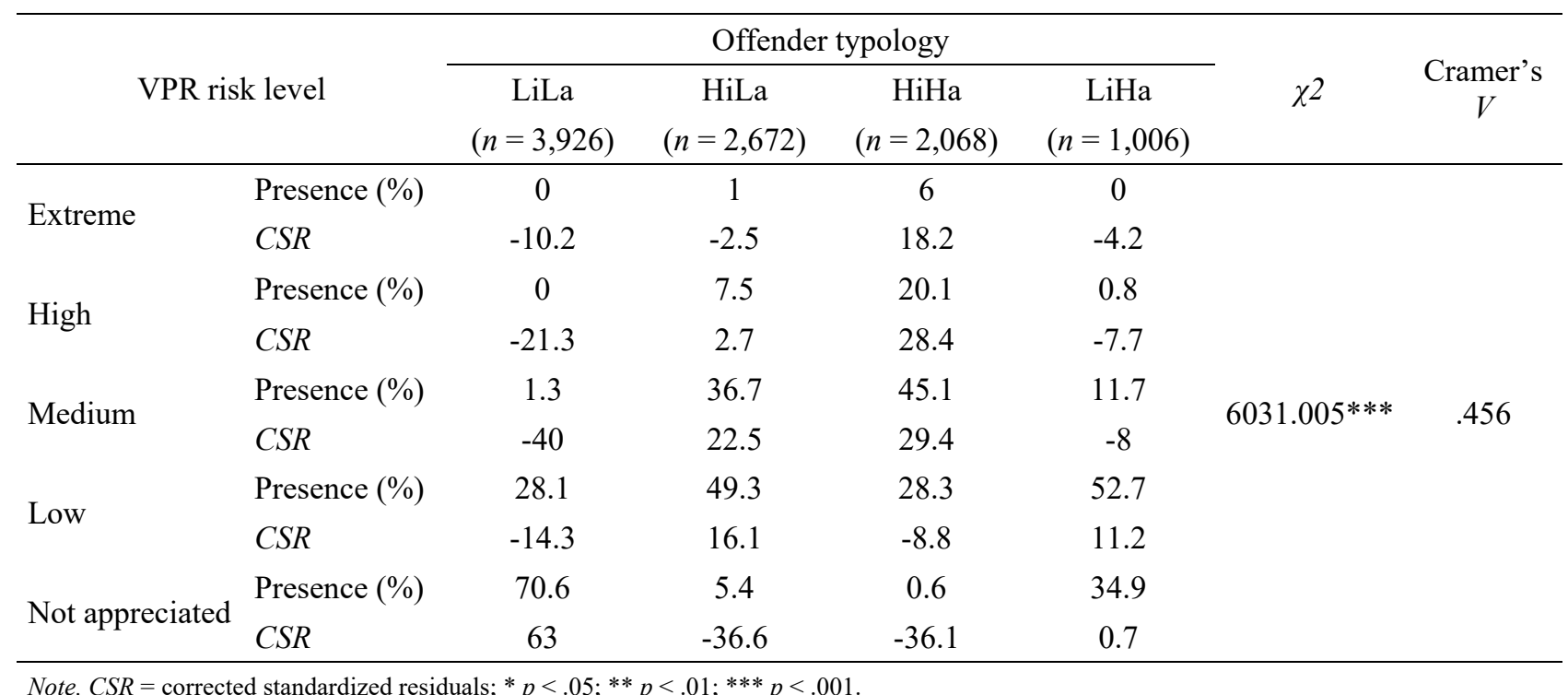


Matching batterer typologies with recidivism risk

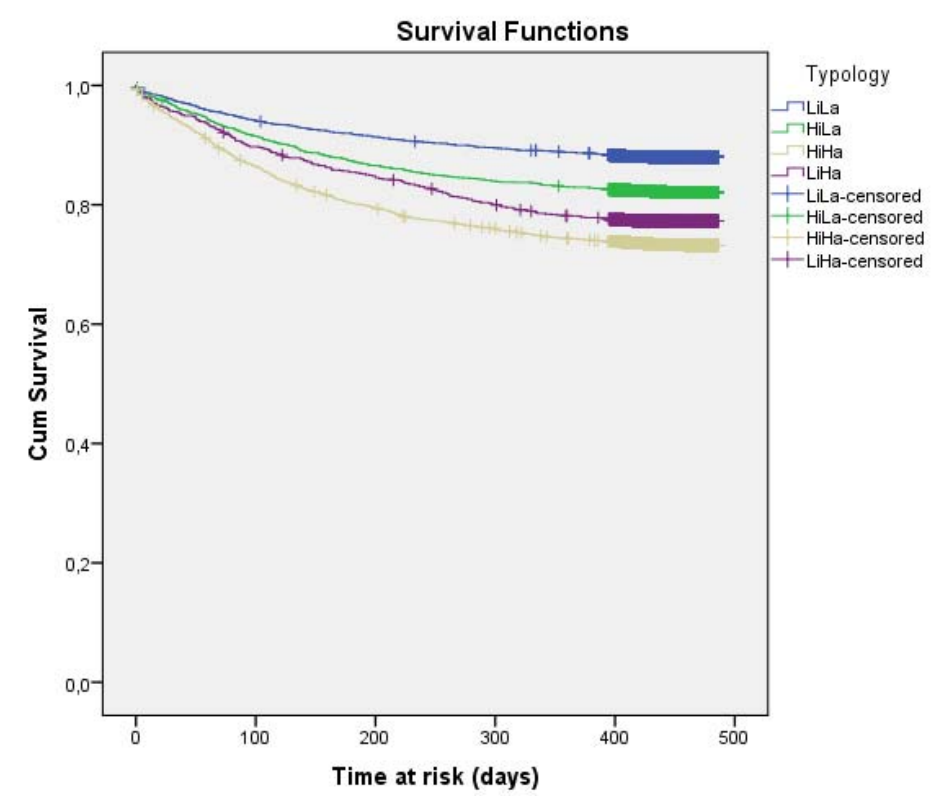

Figure 1. Kaplan-Meier survival plot for police recidivism distinguishing among IPVAW offender subtypes 


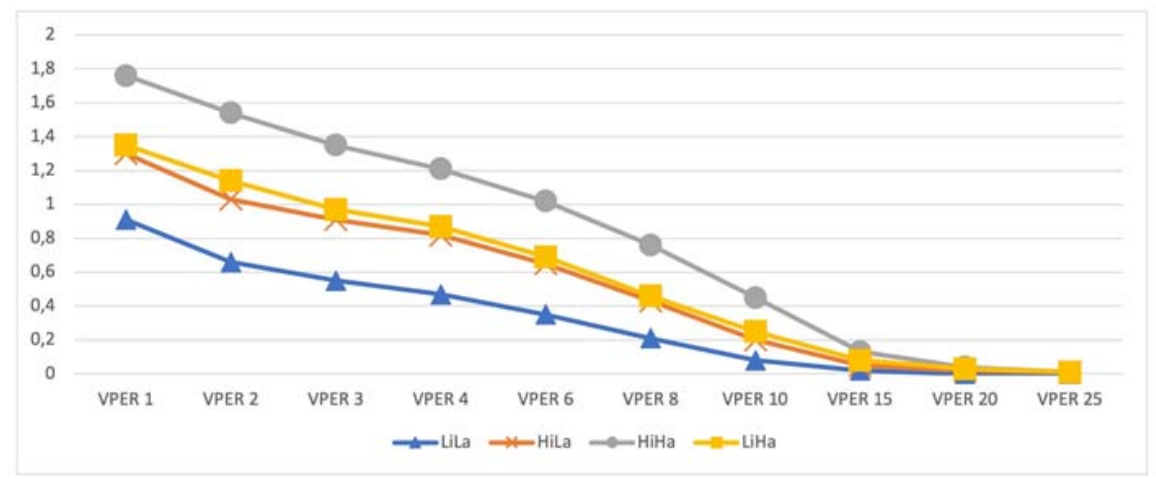

Figure 2. Evolution of recidivism risk levels over time for each IPVAW offender subtype

' IPVAW is legally referred to as gender-based violence in Spain (see Organic Act 1/2004 of 28 December on Integrated Protection Measures against Gender Violence), and is considered the manifestation of discrimination, the situation of inequality and the power relations of men over women, exercised over them by those who are or have been their spouses or who are or have been linked to them by similar relationships of affectivity, even without cohabitation.

ii Catalonia is the only Spanish Autonomous Community that gathers all the penitentiary administration competences transferred. The prison population in Catalonia represents $14.3 \%$ of the national total (Ministerio del Interior, 2020).

iii The suspension of prison sentences is contemplated by the Spanish legislation for cases in which custodial sentences do not exceed two years, in non-habitual offenders. The alternative measures/penalties to prison are managed by the Alternative Measures and Penalties Management Services. 